\title{
Narrow Passage Sampling for Probabilistic Roadmap Planning
}

\author{
Zheng Sun, Member, IEEE, David Hsu, Tingting Jiang, Hanna Kurniawati, and John H. Reif, Fellow, IEEE
}

\begin{abstract}
Probabilistic roadmap (PRM) planners have been successful in path planning of robots with many degrees of freedom, but sampling narrow passages in a robot's configuration space remains a challenge for PRM planners. This paper presents a hybrid sampling strategy in the PRM framework for finding paths through narrow passages. A key ingredient of the new strategy is the bridge test, which reduces sample density in many unimportant parts of a configuration space, resulting in increased sample density in narrow passages. The bridge test can be implemented efficiently in high-dimensional configuration spaces using only simple tests of local geometry. The strengths of the bridge test and uniform sampling complement each other naturally. The two sampling strategies are combined to construct the hybrid sampling strategy for our planner. We implemented the planner and tested it on rigid and articulated robots in 2-D and 3-D environments. Experiments show that the hybrid sampling strategy enables relatively small roadmaps to reliably capture the connectivity of configuration spaces with difficult narrow passages.
\end{abstract}

Index Terms-Motion planning, probabilistic roadmap (PRM) planner, random sampling, randomized algorithm, robotics.

\section{INTRODUCTION}

D URING the past decade, probabilistic roadmap (PRM) planning [1]-[10] has emerged as a powerful framework for path planning of robots with many degrees of freedom (DOFs). The main idea of a classic PRM planner [7] is to sample at random a robot's configuration space, and connect the sampled points to construct a roadmap graph that captures the connectivity of the free space, the collision-free subset of the configuration space. Due to its efficiency and simplicity, PRM planners have found many applications in addition to robotics, including virtual prototyping and computational biology (see, e.g., [11]-[15]).

Manuscript received January 3, 2005. This paper was recommended for publication by Associate Editor G. Oriolo and Editor S. Hutchinson upon evaluation of the reviewers' comments. This work was supported in part by the National Science Foundation (NSF) ITR under Grants EIA-0086015 0326157 and CCR-0086013, in part by the NSF QuBIC under Grants EIA-0218376 and EIA-0218359, in part by the Defense Advanced Research Projects Agency/Air Force Office of Scientific Research under Contract F30602-01-2-0561, in part by the Research Grants Council under Grant HKBU2107/04E, and in part by the National University of Singapore (NUS) under Grant R252-000-154-112/101. This paper was presented in part at the International Conference on Robotics and Automation, Taipei, Taiwan, R.O.C., September 2003.

Z. Sun is with the Department of Computer Science, Hong Kong Baptist University, Kowloon, Hong Kong (e-mail: sunz@comp.hkbu.edu.hk).

D. Hsu and H. Kurniawati are with the Department of Computer Science, National University of Singapore, Singapore 117543, Singapore (e-mail: dyhsu@comp.nus.edu.sg; hannakur@comp.nus.edu.sg).

T. Jiang and J. H. Reif are with the Department of Computer Science, Duke University, Durham, NC 27708 USA (e-mail: ruxu@cs.duke.edu; reif@cs.duke.edu).

Digital Object Identifier 10.1109/TRO.2005.853485
Despite the success of PRM planners, path planning for multiple-DOF robots is difficult. Several instances of the problem have been proven to be PSPACE-hard [16] or even undecidable [17], [18]. It is unlikely that sampling, the key idea behind PRM planners, can overcome such difficulty entirely. Indeed, narrow passages in the configuration space pose significant difficulty for PRM planners. Intuitively, narrow passages are small regions whose removal changes the connectivity of the configuration space. We can also give formal characterizations [6], [19] using the notion of visibility sets, where two points in the configuration space are considered visible to each other if they can be connected by a collision-free straight-line path. To capture the connectivity of the configuration space well, PRM planners must sample in the narrow passages. This is difficult, because narrow passages have small volumes, and the probability of sampling from small sets is low.

In this paper, we propose a hybrid sampling strategy in the PRM framework in order to find paths through narrow passages efficiently. Our goal is to build a good roadmap by sampling a small number of well-placed points from the configuration space. We pay a slightly higher computational cost in sampling than simpler alternatives, but our roadmap requires much fewer points to capture the connectivity of the configuration space, thus saving a lot of time in checking collision-free connections between the sampled points.

A key ingredient of our new strategy is the bridge test, a specialized sampling strategy for narrow passages. It rejects a large fraction of samples in unimportant parts of a configuration space, thus resulting in increased sample density in narrow passages. In a bridge test, we check for collision at three sampled points: the two endpoints and the midpoint of a short line segment $s$. If the two endpoints are in collision and the midpoint is collision-free, the midpoint is accepted as a new node in the roadmap graph being constructed. We call this a bridge test, because the line segment $s$ resembles a bridge: the endpoints of $s$, located inside obstacles, act as piers, and the midpoint hovers over the free space.

The bridge test saves computation time by filtering out those sampled points that are unlikely to contribute to an improved roadmap. For a point inside a narrow passage, building short bridges through it is easy, due to the geometry of narrow passages; for a point in the middle of wide-open free space, doing so is much more difficult. By favoring short bridges, we filter out many sampled points in wide-open free space, as most of these points do not improve the connectivity of the roadmap. At the same time, points inside narrow passages easily pass the bridge test and are retained in the roadmap to improve its connectivity. See Fig. 1. 


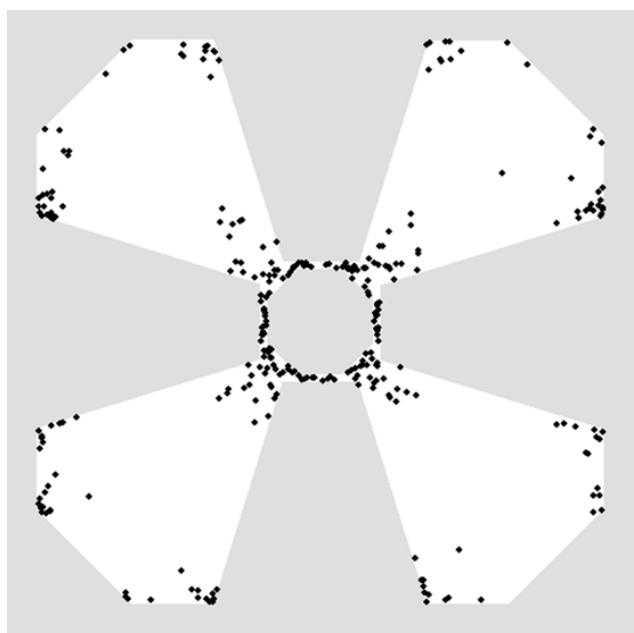

Fig. 1. Example of sample points generated with the bridge test. In this and all later figures, shaded regions indicate obstacles. Black dots indicate sample points.

The bridge test uses only collision checking as a primitive operation, and does not require complex geometric processing in the configuration space. It is simple to implement and can be easily applied to high-dimensional configuration spaces.

Since the bridge test focuses almost solely on the narrow passages, it may fail to sample an adequate number of points to cover the entire free space [19]. Interestingly, the difficulty encountered by the bridge test can be overcome by uniform sampling, which tends to place many samples in wide-open free space. The strengths of the bridge test and the uniform sampler complement each other naturally, and the two sampling strategies are combined to produce a hybrid sampling strategy to achieve better results.

In the following, Section II reviews related work. Section III gives an overview of our planner. Sections IV and V present the bridge test and show how to combine it with uniform sampling to construct a hybrid sampling strategy. Section VI discusses practical implementation issues. Section VII reports experiments with our planner on rigid and articulated robots in 2-D and 3-D environments. Section VIII discusses the limitation of the bridge test and possible generalization. Section IX summarizes the main results.

\section{RELATED WORK}

The difficulty posed by narrow passages and its importance were noted in early work on PRM planners (see, e.g., [7]) and were later articulated in [20]. Several sophisticated sampling strategies can alleviate this difficulty, but a satisfactory answer remains elusive.

One possibility is to sample more densely near obstacle boundaries [1], [3], [21], because points in narrow passages lie close to obstacles. The Gaussian sampler [3] is a simple, efficient algorithm that uses this idea. However, in some cases, many points near obstacle boundaries lie far away from narrow passages and do not help in improving the connectivity of roadmaps. So despite the improvement, sampling near obstacle boundaries may waste many samples in uninteresting regions. In some special cases, the Gaussian sampler can be extended to reduce the number of wasted samples by paying a higher computational cost.

Other approaches to narrow passage sampling include dilating the free space [20] and retracting to the medial axis of free space [22]. Both require geometric operations that are expensive to implement in high-dimensional configuration spaces. To reduce the computational cost, various approximation techniques have been proposed [5], [23], [24]. The visibility-based PRM [10] is related to the narrow passage problem. It tries to reduce the number of unnecessary roadmap nodes by checking their visibility.

Some of the above approaches and others are compared through systematic experiments [25], [26]. The comparison indicates that the various approaches all have their own strengths for different situations. Thus, it is natural to combine them [27]-[29]. This idea, which we call hybrid sampling, is also used here.

\section{OVERVIEW OF THE PlanNER}

The configuration of a robot with $n$ DOFs can be represented as a point in an $n$-dimensional space $\mathcal{C}$, called the configuration space. A configuration $q$ is free if the robot placed at $q$ does not collide with the obstacles or with itself. We define the free space $\mathcal{F}$ to be the set of all free configurations in $\mathcal{C}$, and define the obstacle space $\mathcal{B}$ to be the complement of $\mathcal{F}: \mathcal{B}=\mathcal{C} \backslash \mathcal{F}$.

A classic multiquery PRM planner proceeds in two stages. In the first stage, it randomly samples in $\mathcal{F}$ a set of points, called milestones. It uses the milestones as nodes to construct a graph $G$, called a roadmap, by adding an edge between every pair of milestones that can be connected via a simple collision-free path, typically, a straight-line segment. After the roadmap has been constructed, multiple queries can be answered quickly in the second stage. Each query consists of an initial configuration $s$ and a goal configuration $t$, and asks for a collision-free path connecting $s$ and $t$. The planner first finds two milestones $s^{\prime}$ and $t^{\prime}$ in the roadmap $G$, such that $s$ (t, respectively) and $s^{\prime}\left(t^{\prime}\right.$, respectively) can be connected by a collision-free path in $\mathcal{C}$, and then searches for a path in $G$ between $s^{\prime}$ and $t^{\prime}$.

In this paper, we follow this general framework, but address mainly the first stage, roadmap construction. Methods for the second stage are well understood [7], [25].

An important property of a good roadmap $G$ is coverage: for any given (initial or goal) configuration $q \in \mathcal{F}$, there is a collision-free path between $q$ and a milestone in $G$ with high probability. This implies that the milestones in $G$ collectively "covers" a significant portion of $\mathcal{F}$. Another important property is connectivity. The roadmap $G$ should capture the connectivity of the underlying free space $\mathcal{F}$ that it represents. Any two milestones in the same connected component of $\mathcal{F}$ should also be connected by a path in $G$. Otherwise, the planner would give many false negative answers.

A main difficulty in constructing good roadmaps results from narrow passages in $\mathcal{F}$. Narrow passages are small regions whose removal changes the connectivity of $\mathcal{F}$ : they may change the way different regions are connected, or even change the number of connected components. To capture the connectivity of $\mathcal{F}$ in the roadmap, it is essential to sample milestones in narrow passages. This, however, is difficult, because of their small volumes. Any volume-based sampling distribution is likely to fail. 
In particular, the uniform distribution does not work well. Furthermore, when dealing with many-DOF robots, we do not have an explicit representation of $\mathcal{F}$ and cannot locate narrow passages directly by processing the global geometry of $\mathcal{F}$.

Our goal is to build a good roadmap by sampling a small number of well-placed milestones. To obtain milestones in narrow passages, we pay a higher cost for sampling a milestone than do simpler methods, such as uniform sampling. However, the intuition is that our roadmap would require much fewer milestones to cover $\mathcal{F}$ and capture its connectivity, thus saving a lot of time in checking collision-free connections between the milestones.

More precisely, the running time $T$ of roadmap construction is given by

$$
T=N_{\text {mil }} \cdot T_{\text {mil }}+N_{\text {con }} \cdot T_{\text {con }}
$$

where

$T_{\text {mil }}$ average cost of sampling a milestone;

$N_{\text {mil }}$ number of milestones in the roadmap;

$T_{\text {con }}$ average cost of checking collision-free connections between two milestones;

$N_{\text {con }}$ number of calls to check collision-free connections between two milestones.

To illustrate the potential benefits of our approach, let us look at a numerical example. Assume that $T_{\mathrm{mil}}=t$ and $T_{\text {con }}=10 t$, as the cost of checking collision-free connections between two milestones is much higher than that of sampling a milestone. Assume also that $N_{\text {mil }}=n$, and every milestone is checked for connection with two nearby milestones, i.e., $N_{\text {con }}=2 \cdot N_{\text {mil }}=$ $2 n$. The total running time is then $T=n \cdot t+2 n \cdot 10 t=21 n t$. Now suppose that we pay a higher cost in sampling, but are able to reduce the number of milestones needed. For example, $T_{\text {mil }}=100 t$, and $N_{\text {mil }}=n / 10$. Then we have the total running time $T=n / 10 \cdot 100 t+2 n / 10 \cdot 10 t=12 n t$, which is roughly half of the original running time. By paying a higher cost $T_{\text {mil }}$, we reduce the number of milestones needed in the roadmap, and thus reduce $N_{\text {con }}$. Since $T_{\text {con }}$ is usually much larger than $T_{\text {mil }}$, the cost of checking connections between milestones dominates, and reducing $N_{\text {con }}$ results in reducing the total running time. Although the numerical values in this example are chosen for the purpose of illustration and should not be taken literally, the intuition behind holds more generally and is supported by the experiments (see Section VII). However, we must be careful in balancing the cost and the benefit. If the increase in $T_{\mathrm{mil}}$ is much higher than the decrease in $N_{\text {con }}$, the benefit of our approach will be diminished. Therefore, $T_{\mathrm{mil}}$ should be kept small, if possible.

The sampling distribution that we use for our planner is a weighted mixture of $\pi_{\mathrm{B}}$, the distribution generated by the bridge test, and $\pi_{\mathrm{U}}$, the uniform distribution. We describe how to construct $\pi_{\mathrm{B}}$ and combine the two distributions in the next two sections.

After sampling a new milestone $q$, our planner tries to connect $q$ to nearby milestones via collision-free straight-line paths. Like other PRM variants, our planner tries to connect two milestones only if they lie in different connected components of the roadmap $G$ and the distance between them is smaller than a threshold, according to a suitable distance metric. However,
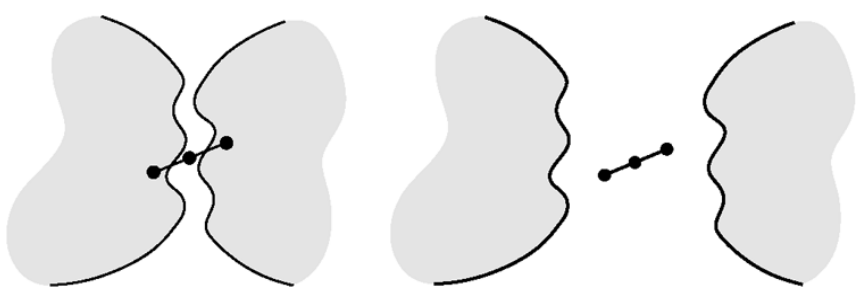

Fig. 2. Building short bridges is much easier in narrow passages (left) than in wide-open free space (right).

\begin{tabular}{l}
\hline Algorithm 1 Randomized Bridge Builder (RBB). \\
\hline 1. repeat \\
2. $\quad$ Pick a point $x$ from $\mathcal{C}$ uniformly at random. \\
3. if CLEARANCE $(x)$ returns FALSE then \\
4. $\quad$ Pick a point $x^{\prime}$ in the neighborhood of $x$ according \\
to a suitable probability density $\lambda_{x}$. \\
5. $\quad$ if CLEARANCE $\left(x^{\prime}\right)$ returns FALSE then \\
7. $\quad$ Set $q$ to be the midpoint of line segment $\overline{x x^{\prime}}$. \\
8. \\
if CLEARANCE $(q)$ returns TRUE then \\
\hline
\end{tabular}

when the size of $G$ is large, there may still be too many milestones whose distances to $q$ are less than the threshold. So the planner tries to connect $q$ with only the $K$ nearest such milestones, where $K$ is a fixed constant. The intuition is that if the planner cannot establish connections from $q$ to the $K$ nearest milestones, most likely it will not be able to establish connections to other milestones further away.

\section{The BRIDGE TEST}

\section{A. The Algorithm}

The bridge test is designed to boost the sample density in narrow passages using only simple tests of local geometry. It is based on the following observation. A narrow passage in an $n$-dimensional configuration space has at least one restricted direction $v$ such that small perturbations of the robot's configuration along $v$ result in collision of the robot with obstacles. Therefore, for a collision-free configuration $q$ in a narrow passage, it is easy to sample at random a short line segment $s$ through $q$, such that the endpoints of $s$ lie in the obstacles in $\mathcal{C}$ (Fig. 2, left). The line segment $s$ is called a bridge, because it resembles a bridge across the narrow passage. We say that a point $q \in \mathcal{F}$ passes the bridge test, if we succeed in obtaining such a segment $s$ through $q$. For convenience, we also say that $s$ passes the bridge test. Clearly, building short bridges is much easier in narrow passages than in wide-open free space. By favoring shorter bridges over longer ones, we increase the chance of accepting points in narrow passages (Fig. 2).

To sample a new milestone using the bridge test, we pick a line segment $s$ from $\mathcal{C}$ by choosing its endpoints at random and testing whether $s$ passes the bridge test. If so, we add the midpoint of $s$ to the roadmap $G$ as a new milestone. We call this algorithm Randomized Bridge Builder (RBB). The details of RBB are shown in Algorithm 1. In lines 3, 5, and 7, RBB calls the function CLEARANCE to test whether a point in $\mathcal{C}$ is collision-free.

To perform the bridge test, RBB uses only a single geometric primitive, CLEARANCE, which can be implemented efficiently 
using a collision detection algorithm (see, e.g., [30], [31]). The bridge test is purely local, and does not require processing the global geometry of $\mathcal{C}$.

RBB pays a higher cost to sample a milestone than simpler alternatives such as uniform sampling, because of two reasons. First, it takes three calls to CLEARANCE for RBB to accept a milestone, one for the milestone itself, and two for the endpoints of the bridge passing through the milestone. Second, it rejects a large fraction of free configurations that are normally accepted by other sampling strategies. However, RBB increases the sample density in narrow passages, which is critical in capturing the connectivity of the free space. For configuration spaces with difficult narrow passages, it usually leads to smaller roadmaps and saves lots of computation time in checking collision-free connections between milestones, an operation that is usually more expensive than the additional cost that RBB incurs in sampling milestones.

\section{B. Choosing the Probability Density $\lambda_{x}$}

The density function $\lambda_{x}$ (Algorithm 1, line 4) determines how frequently a bridge of particular length is chosen for a test at the point $x$. Short bridges are preferred over longer ones in order to increase the probability of sampling in narrow passages. We choose $\lambda_{x}$ to be a radially symmetric Gaussian with its center at $x$ and a small standard deviation $\sigma$. To be specific, let $N(\sigma)$ denote the univariate Gaussian distribution with mean 0 and standard deviation $\sigma$. We sample a value randomly and independently for each dimension of $\mathcal{C}$ according to $N(\sigma)$, shifted to center at the corresponding coordinate of $x$. The density function $\lambda_{x}$ is then the product of these independent univariate Gaussians. Other ways to construct a radially symmetric Gaussian are also possible. Finally, the parameter $\sigma$ depends on the width of narrow passages that we want to capture. The best value for $\sigma$ is problem-specific, and we discuss this issue further in Section VI-B.

\section{Analysis of the Sampling Distribution}

To calculate probability density $\pi_{\mathrm{B}}$ of the milestones created by Algorithm 1, let us first define $X$ and $X^{\prime}$ to be two random variables, representing, respectively, the two endpoints of a bridge. The first endpoint $X$ is distributed uniformly over the set of configuration-space obstacles $\mathcal{B}$. So the density $f_{X}(x)$ is nonzero if and only if $x$ lies in $\mathcal{B}$. Assume, without loss of generality, that $\mathcal{B}$ has volume 1 . Then $f_{X}(x)$ is 1 if $x \in \mathcal{B}$, and 0 otherwise. Given $X=x$, we choose the other endpoint $X^{\prime}=x^{\prime}$ according to the density $\lambda_{x}$. The point $x^{\prime}$ is accepted only if it lies in $\mathcal{B}$. Let $I$ be a binary function such that for any point $q \in \mathcal{C}, I(q)=1$ if $q \in \mathcal{B}$, and 0 otherwise. The conditional density of $X^{\prime}$ given $X$ is given by

$$
f_{X^{\prime} \mid X}\left(x^{\prime} \mid x\right)=\lambda_{x}\left(x^{\prime}\right) I\left(x^{\prime}\right) / Z_{x}
$$

where $Z_{x}=\int_{\mathcal{C}} \lambda_{x}\left(x^{\prime}\right) I\left(x^{\prime}\right) d x^{\prime}$ is a normalizing constant. To calculate $\pi_{\mathrm{B}}$ at a point $q \in \mathcal{F}$, we condition on $X$

$$
\pi_{\mathrm{B}}(q)=\int_{\mathcal{C}} f_{X^{\prime} \mid X}\left(x^{\prime} \mid x\right) f_{X}(x) d x .
$$

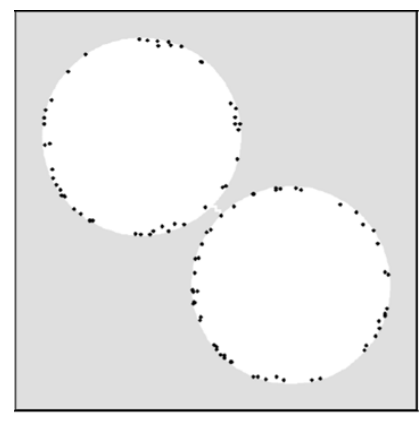

Gaussian sampler

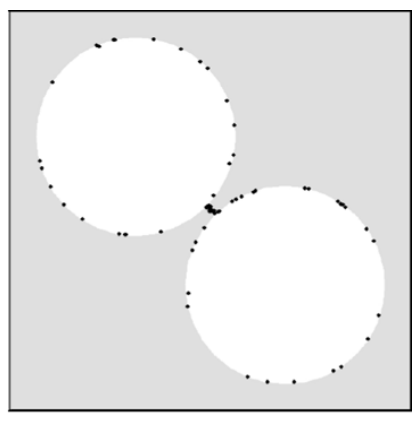

$\mathrm{RBB}$
Fig. 3. Milestones generated by the Gaussian sampler and RBB. The total number of milestones in the two cases is the same. RBB generates fewer milestones along the circular boundaries and many more in the narrow passage connecting the two circular chambers.

Note that $p$ is the midpoint of the line segment $\overline{x x^{\prime}}$, and so $x^{\prime}=$ $2 q-x$. Substituting the expressions for $f_{X}, f_{X^{\prime} \mid X}$, and $x^{\prime}$ into (1), we have

$$
\pi_{\mathrm{B}}(q)=\int_{\mathcal{B}} \lambda_{x}(2 q-x) I(2 q-x) / Z_{x} d x .
$$

We have chosen $\lambda_{x}$ to be a Gaussian with its center at $x$ and a small standard deviation. The density $\lambda_{x}$ is large if $x^{\prime}=2 q-x$ lies relatively close to $x$. Furthermore, the integrand in (1) is nonzero only if $I(2 q-x)=1$, i.e., $x^{\prime} \in \mathcal{B}$. In the neighborhood of a point $q$ inside a narrow passage, it is more likely to find pairs of points $x$ and $x^{\prime}$ that satisfy these two conditions, resulting in a larger value for $\pi_{\mathrm{B}}$ at $q$.

\section{Comparison With Sampling Near Obstacle Boundaries}

$\mathrm{RBB}$ is related to the Gaussian sampler [3]. Both use one simple geometric primitive CLEARANCE to create favorable distributions. RBB is slightly more expensive: it makes one more call to CLEARANCE in each invocation than the Gaussian sampler, and rejects more samples. However, the nature of the two sample distributions generated are quite different. RBB increases the sample density in regions where short bridges can be easily constructed; the Gaussian sampler increases the sample density near obstacle boundaries. See Fig. 3 for the difference between the two sample distributions. If milestones near obstacle boundaries all improve the connectivity of roadmaps, the Gaussian sampler is preferable, as it incurs lower cost per invocation. On the other hand, obstacle boundaries may be uninteresting if they are bounding wide-open regions of $\mathcal{F}$, as samples near these boundaries do not contribute to improving the connectivity of roadmaps. Therefore, RBB gains efficiency by avoiding sampling near such boundaries. In this sense, RBB and the Gaussian sampler are complementary.

\section{COMBINING COMPLEMENTARY SAMPLING DisTRIBUTIONS}

We have seen that RBB helps in boosting the sample density in the subset $\mathcal{P}$ of $\mathcal{F}$ occupied by narrow passages. The density $\pi_{\mathrm{B}}$ is heavily biased toward $\mathcal{P}$. At the same time, $\pi_{\mathrm{B}}$ penalizes wide-open collision-free regions: few milestones are sampled in $\mathcal{F} \backslash \mathcal{P}$. This is undesirable, because a good roadmap must cover the entire free space adequately. 


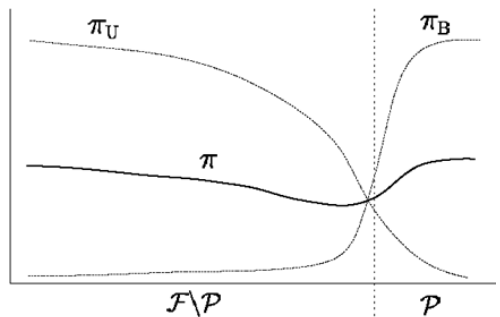

Fig. 4. Hybrid sampling distribution $\pi$. The distributions $\pi_{\mathrm{B}}$ and $\pi_{\mathrm{U}}$ perform well on $\mathcal{P}$ and $\mathcal{F} \backslash \mathcal{P}$, respectively. Combining them with suitable weights leads to good performance over the entire sampling domain $\mathcal{F}$.

Interestingly, we can make up the deficiency of $\pi_{\mathrm{B}}$ with the uniform distribution $\pi_{\mathrm{U}}$, which samples $\mathcal{F}$ with probability proportional to the volumes of subsets in $\mathcal{F}$. For $\pi_{\mathrm{U}}$, most milestones are sampled in $\mathcal{F} \backslash \mathcal{P}$, as it has a large volume. The two distributions complement each other naturally: $\pi_{\mathrm{U}}$ provides good coverage of $\mathcal{F} \backslash \mathcal{P}$, and $\pi_{\mathrm{B}}$ samples more densely in $\mathcal{P}$, and thus improves the connectivity of the roadmap. They are combined to produce a hybrid sampling distribution

$$
\pi=(1-w) \cdot \pi_{\mathrm{B}}+w \cdot \pi_{\mathrm{U}}
$$

where $w$ is a weight, with $0 \leq w \leq 1$. The choice of $w$ depends on the difficulty of sampling in narrow passages and the number of milestones needed to cover $\mathcal{F}$. One useful way of thinking about this hybrid distribution $\pi$ is to divide $\mathcal{F}$ into two subsets, the set of narrow passages $\mathcal{P}$ and its complement $\mathcal{F} \backslash \mathcal{P}$. We use a different sampling strategy tailored to each subset, and combine them to achieve good performance over the entire sampling domain. See Fig. 4 for an illustration. Note, however, that in our planner, the combination of two sampling strategies is achieved through weighting, and not through explicit decomposition of $\mathcal{F}$.

The significance of hybrid sampling is not about putting together two sampling distributions, but rather about identifying distributions complementary in their strengths and combining them so that their individual strengths are preserved.

To implement the hybrid distribution, we can certainly generate new random points from $\pi_{\mathrm{U}}$, but we can get some of these points "for free" by reusing the points rejected by RBB. In line 3 of Algorithm 1, RBB rejects a configuration $x$ if CLEARANCE $(x)$ returns TRUE, i.e., $x$ is collision-free. However, such a configuration $x$ is exactly what is generated by $\pi_{\mathrm{U}}$. To reduce computation time, we can save $x$ and use it instead of generating a new one from $\pi_{\mathrm{U}}$ when needed.

\section{IMPLEMENTATION ISSUES}

In this section, we describe some details for implementing our planner on rigid and articulated robots.

\section{A. Parameterizing the Configuration Space}

Often, each dimension of the configuration space may have a different effect on the overall motion of a robot. Consider, for example, a planar articulated robot with a free base and three links of lengths $l_{1}, l_{2}$, and $l_{3}$, respectively. The configuration of this robot can be represented as $\left(x, y, \theta_{1}, \theta_{2}, \theta_{3}\right)$, where $x$ and $y$ specify the position of the base, and $\theta_{1}, \theta_{2}$, and $\theta_{3}$ specify the angles for the three joints in increasing order of their distance to the base along the kinematic chain. Assume that the robot is fully extended. If $\theta_{1}$ changes by an angle $\varphi$, the tip of the robot moves a distance of $\left(l_{1}+l_{2}+l_{3}\right) \varphi$. If $\theta_{2}$ changes by the same angle $\varphi$, the tip moves by a distance of $\left(l_{2}+l_{3}\right) \varphi$. We must take this into account for our planner in two occasions.

First, when choosing the density function $\lambda_{x}$ in RBB, we need a consistent measure of how restrictive the allowable motion is in the narrow passages along each dimension of the configuration space $\mathcal{C}$. Our solution is to rescale $\mathcal{C}$. For each configuration space coordinate $q_{i}$, let $d_{i}$ be the maximum distance traveled by any point on the robot when the robot moves between any two configurations that have identical coordinates in all dimensions except $q_{i}$ [32]. We rescale the range of $q_{i}$ to $\left[0, d_{i}\right]$. This method of rescaling is quite general, and can be applied to any translational or rotational DOF of a robot. In our planar articulated robot example, for translational DOFs, the scaling factors are 1 , and rescaling has no net effect. For rotational DOFs, we have, for example, $d_{2}=\left(l_{2}+l_{3}\right) 2 \pi$ for the joint angle $\theta_{2}$, and we can scale other joint angles similarly. For rigid robots in 3-D, rotational DOFs can be represented with either Euler angles or quaternions. If Euler angles are used, the scaling factor for each Euler angle is calculated exactly the same way as that for the joint angle of articulated robots: $d_{i}=2 \pi L_{i}$, where $L_{i}$ is the maximum distance of any point on the robot to the axis of rotation for the corresponding Euler angle $q_{i}$. If quaternions are used, the calculation is slightly more involved, but the principle is the same.

We need to consider this scaling issue again when adding a new milestone $q$ to the roadmap. The planner tries to connect $q$ with existing milestones within a certain distance. So we need a suitably defined distance metric on $\mathcal{C}$. Ideally, the metric has the property that for any two free configurations $q$ and $q^{\prime}$, the greater the distance between $q$ and $q^{\prime}$ is, the more likely that the robot encounters an obstacle when following a straight-line path from $q$ to $q^{\prime}$. Again, we have to rescale $\mathcal{C}$, so that each dimension of $\mathcal{C}$ has a similar effect on the overall motion of the robot. After rescaling, we can simply define the metric on $\mathcal{C}$ to be the Euclidean distance between two configurations. This scaling heuristic is often used in motion planning (see, e.g., [7]). Other heuristics can also be used. For instance, instead of using the maximum distance traveled by any point on the robot as a measure of the "distance" between two configurations, we can use the volume swept out by the robot.

\section{B. Parameters for the Sampling Distribution}

Two parameters are needed to fix the hybrid sampling distribution $\pi$. The first parameter fixes the density function $\lambda_{x}$ for RBB. As we have discussed earlier, $\lambda_{x}$ is a product of independent Gaussians, with a small standard deviation $\sigma$ to bias toward sampling short bridges. The parameter $\sigma$ depends on the width of narrow passages to be captured, and may affect the planner's performance. In practice, we can estimate the value of $\sigma$ by analyzing the geometry of the robot and the obstacles in the environment. For example, if the smallest passage in the workspace has width $W$, and a rigid robot, which translates and rotates, has maximum radius $R$, then $\sigma$ can be set to be proportional to $W / R$. 


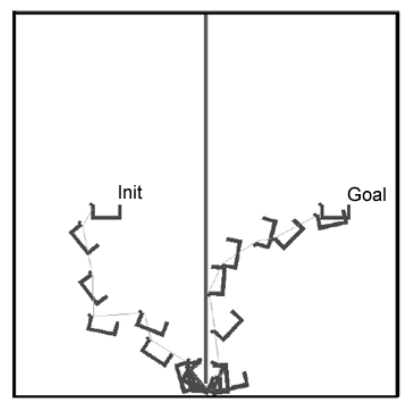

(a)

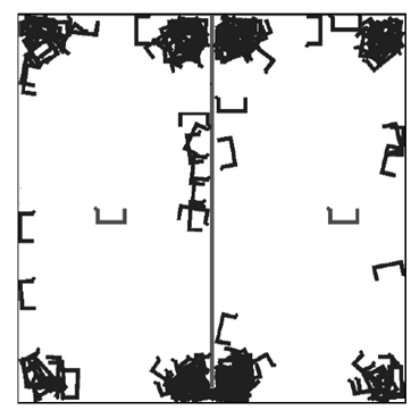

(c)

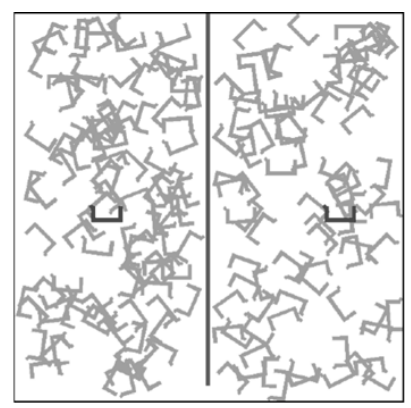

(b)

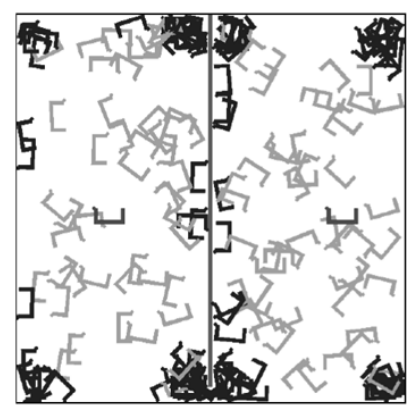

(d)
Fig. 5. Planar rigid-body robot maneuvering through a small opening between two large chambers. (a) Query configurations and a path found. (b) Uniform sampling. (c) RBB. (d) Hybrid sampling.

The second parameter is the weight for combining $\pi_{\mathrm{B}}$ and $\pi_{\mathrm{U}}$. For our experiments, we assume no prior knowledge of the environment. We do not bias toward either $\pi_{\mathrm{B}}$ or $\pi_{\mathrm{U}}$, and use a relative weight of $1: 1$.

The best parameters settings are clearly problem-dependent, and it is difficult to choose them in advance. A promising approach is to use a set of RBBs with different $\sigma$ values and adjust the weight for each RBB adaptively through on-line learning [28]. This way, the best parameter values can be identified automatically in an adaptive way.

\section{EXPERIMENTS}

We implemented two versions of our planner, one version in Java for robots in 2-D environments, and one version in $\mathrm{C}++$ for robots in 3-D environments. To examine the planner's performance, we handcrafted several difficult environments and tested our implementations extensively. The 2-D test environments are described below.

- Fig. 5(a): We have a rigid-body robot, and the query asks the robot to go from one large empty chamber to another through a small opening near the lower middle of the figure. This is a case where we expect the hybrid sampling strategy to work well, because the bridge test eliminates many sampled configurations that lie in the middle of wide-open space and are unlikely to improve the coverage or connectivity of the roadmap. Fig. 5(b)-(d) shows the roadmaps generated by uniform sampling, pure RBB, and hybrid sampling, respectively. Each sampling strategy uses the same number of milestones. The roadmap constructed by uniform sampling covers the free space well, but wastes many milestones

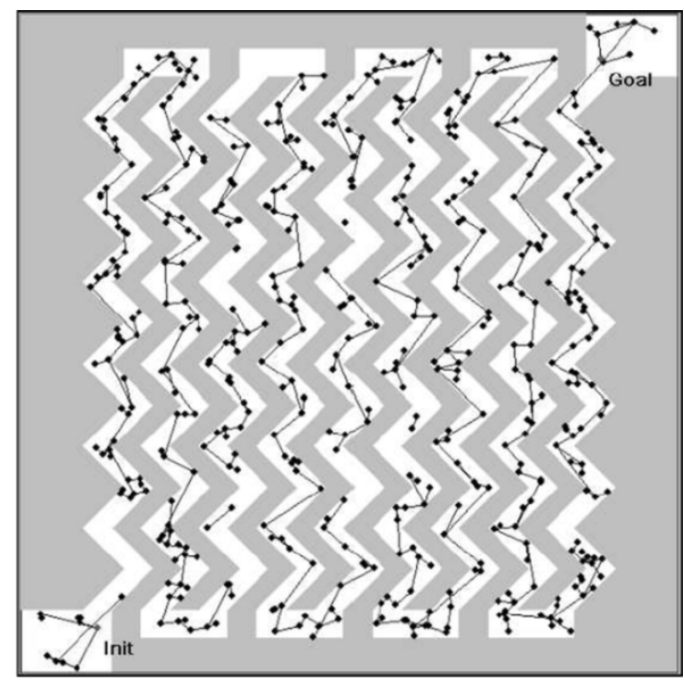

Fig. 6. Partial roadmap built by RBB for a point robot in the plane.

in the two chambers, and does not put any milestone near the small opening. Note that in these figures, the milestones are projected from the 3-D configuration space to the 2-D workspace for drawing. Two milestones that appear close to each other in the figures may be far away from each other in the configuration space because of different orientations, and therefore, there may not be a collision-free straight-line path that connects them. Pure RBB puts a large number of milestones near places where it can successfully build "bridges," including the small opening between chambers. This enables it to capture the connectivity of the free space well. However, the roadmap constructed by RBB has poor coverage: very few milestones are in the two chambers to cover the free space. This does not cause a big problem here, because the two chambers in this example are convex, and every point inside a chamber can cover a large portion of the chamber. However, in general, more milestones are needed, if the chambers have more complex geometry. Hybrid sampling combines the strengths of uniform sampling and RBB to build a roadmap that has good coverage and captures the connectivity of the free space well.

- Fig. 6: According to our analysis in Section III, sampling with the bridge test works well only if the resulting roadmap contains much fewer milestones than those generated by other sampling strategies. We constructed this example so that the bridge test does not have such an advantage. This planar environment for a point robot contains a long path that has almost equal width everywhere, so almost every sampled configuration passes the bridge test. The roadmap constructed with the bridge test would have roughly the same size as that constructed by uniform sampling or Gaussian sampling.

- Fig. 7: A rigid-segment robot enters a narrow corridor, reorients in a small circular room, and exits another narrow corridor. This environment is an example of connected narrow passages in different orientations. When the robot is inside one of the two corridors, it 


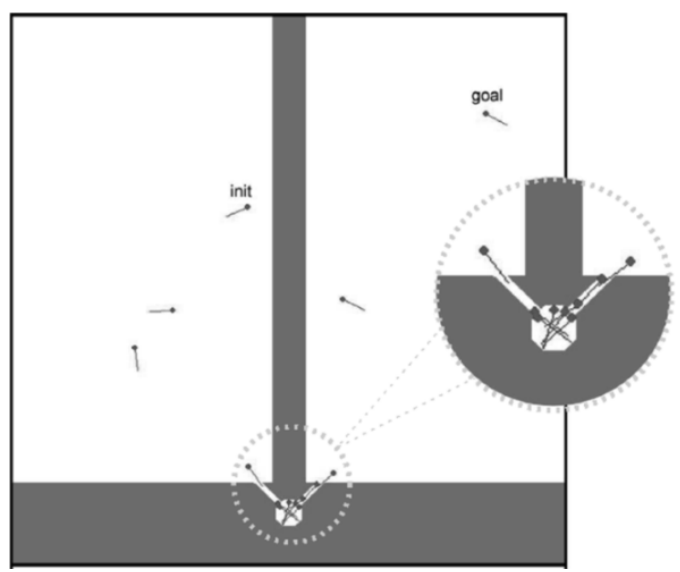

Fig. 7. Path for a rigid segment translating and rotating in a narrow region.

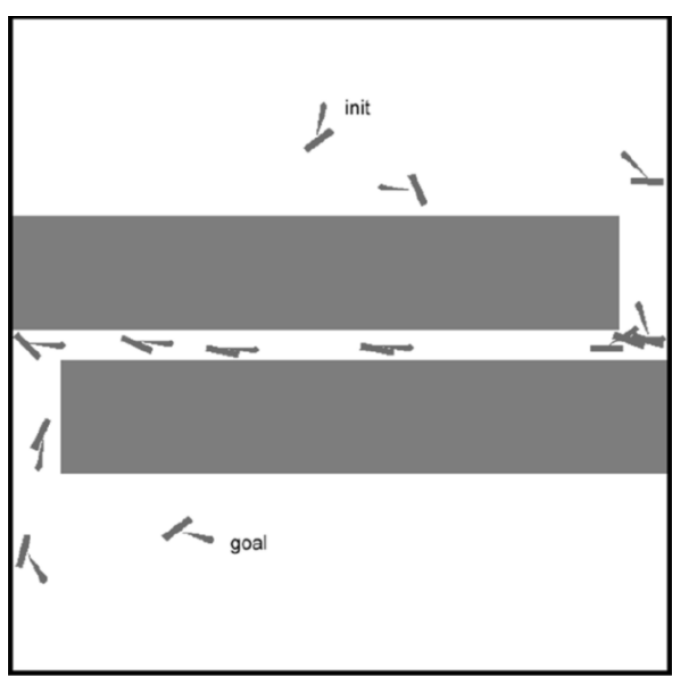

Fig. 8. Path for a T-shaped robot moving through a long, narrow corridor.

can only translate along the direction of the corridor. Movements in the orthogonal directions are very restricted: it cannot translate sidewise or rotate. When the robot is inside the small circular room in the middle, the rotational movement is unrestricted, but the translational movement is very restricted, as the diameter of the room is only slightly more than the length of the robot. In the 3-D configuration space, the two corridors and the round room in the middle map to three connected narrow passages in different orientations.

- $\quad$ Fig. 8: In this example, we have a T-shaped robot with two parts, a "torso" and a "shoulder," connected by a joint. The planar environment contains a long and narrow corridor with two turns.

- $\quad$ Fig. 9: This environment contains a 7-DOF articulated robot with a fixed base. At the initial configuration, the robot is trapped inside a narrow opening. Joint angles near the robot's base have a very limited range of motion. Joint angles near the robot's tip can move relatively freely. As the robot performs difficult maneuvers to pull out of the narrow opening, an increasing number of joints have their motion restricted, until the robot pulls out completely and all the joints can move freely. The robot

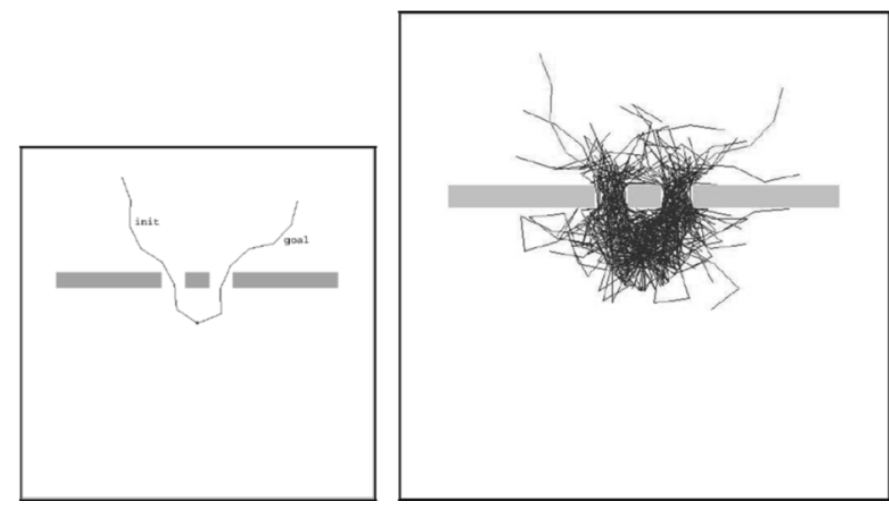

Fig. 9. 7-DOF articulated robot with a fixed base. The left figure shows the query configurations. The right figure shows those milestones generated by RBB.

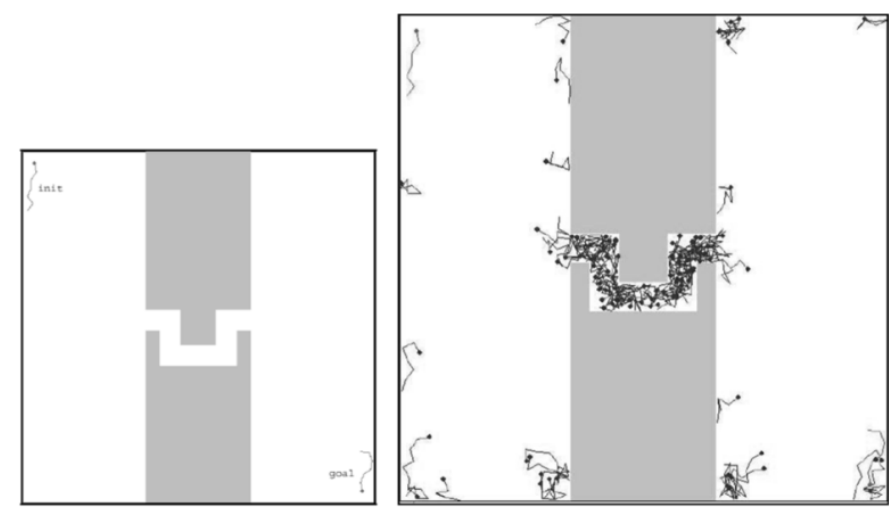

Fig. 10. 8-DOF articulated robot with a mobile base. The left figure shows the query configurations. The right figure shows those milestones generated by RBB.

then has to insert itself into the other narrow opening to reach the goal configuration. The sequence of events are similar, but occur in reverse order.

- Fig. 10: This environment contains a relatively short corridor with two turns. Each milestone in the corridor covers only a small portion of the free space. The robot is an articulated robot with six links and a mobile base, 8 DOFs in total.

We tested these environments with the Java implementation of our planner. For each test environment, we rescaled the configuration space $\mathcal{C}$ to $\left[0, d_{1}\right] \times\left[0, d_{2}\right] \times \cdots$, as described in Section VI. Since all the environments are bounded, we applied an additional uniform scaling $1 / d$, where $d=\max _{i} d_{i}$, so that $\mathcal{C}$ fits within a unit hypercube, in order to simplify the implementation.

The relative weight for combining the two components $\pi_{\mathrm{U}}$ and $\pi_{\mathrm{B}}$ of the hybrid sampling distribution was $1: 1$, i.e., equal weights for the two components. We also systematically varied the standard deviation $\sigma$ of the Gaussian for the bridge test between $1 / 128$ and $1 / 16$ to choose the best setting. It turned out that $\sigma=1 / 32$ worked well in all the experiments.

To add a new milestone $q$ to the roadmap, the planner tries to connect $q$ with an existing milestone $q^{\prime}$, only if: 1) $q$ and $q^{\prime}$ are in different connected components; 2 ) the distance between $q$ and $q^{\prime}$ is smaller than a threshold $D$; and 3) $q^{\prime}$ is a $K$-nearest neighbor of $q$. This method of adding nodes, called nearest- $K$, 
TABLE I

PERFORMANCE OF VARIOUS SAMPLING STRATEGIES

\begin{tabular}{|c|c|c|c|c|c|}
\hline \multirow{2}{*}{$\begin{array}{l}\text { Env. } \\
\text { (Fig.) }\end{array}$} & \multirow[t]{2}{*}{ dofs } & \multirow[t]{2}{*}{ Sampler } & \multirow[t]{2}{*}{$N_{\mathrm{mil}}$} & \multicolumn{2}{|c|}{ Time (sec.) } \\
\hline & & & & avg. & std. \\
\hline \multirow[t]{5}{*}{5} & 3 & Hybrid & 302 & 3.9 & 2.2 \\
\hline & & $\mathrm{RBB}$ & 4,555 & 30.0 & 48.1 \\
\hline & & Uniform & 25,607 & 382.4 & 350.6 \\
\hline & & Visibility & 83 & 120.3 & 96.5 \\
\hline & & Gaussian & 1,165 & 5.6 & 3.2 \\
\hline \multirow[t]{5}{*}{7} & 3 & Hybrid & 900 & 7.4 & 2.8 \\
\hline & & $\mathrm{RBB}$ & 5,956 & 34.7 & 25.9 \\
\hline & & Uniform & 47,064 & 995.0 & 234.7 \\
\hline & & Visibility & 108 & 235.9 & 106.8 \\
\hline & & Gaussian & 2,276 & 8.4 & 3.9 \\
\hline \multirow[t]{5}{*}{8} & 4 & Hybrid & 916 & 7.1 & 2.9 \\
\hline & & RBB & 3,422 & 17.1 & 6.3 \\
\hline & & Uniform & 23,152 & 354.0 & 204.3 \\
\hline & & Visibility & 209 & 148.8 & 45.3 \\
\hline & & Gaussian & 1,976 & 9.9 & 3.8 \\
\hline \multirow[t]{5}{*}{9} & 7 & Hybrid & 1,307 & 111.3 & 42.1 \\
\hline & & RBB & 2,915 & 231.1 & 85.5 \\
\hline & & Uniform & 22,465 & 1012.3 & 380.6 \\
\hline & & Visibility & 826 & 790.6 & 288.5 \\
\hline & & Gaussian & 1,619 & 146.5 & 54.3 \\
\hline \multirow[t]{5}{*}{10} & 8 & Hybrid & 1,004 & 41.4 & 13.3 \\
\hline & & RBB & 4,155 & 98.2 & 26.2 \\
\hline & & Uniform & 35,822 & 1803.3 & 783.2 \\
\hline & & Visibility & 620 & 698.1 & 173.8 \\
\hline & & Gaussian & 3,255 & 92.2 & 27.6 \\
\hline \multirow[t]{5}{*}{6} & 2 & Hybrid & 1,317 & 25.6 & 6.3 \\
\hline & & RBB & 1,390 & 26.4 & 5.5 \\
\hline & & Uniform & 1,358 & 23.4 & 5.4 \\
\hline & & Visibility & 206 & 42.6 & 11.5 \\
\hline & & Gaussian & 1,291 & 23.5 & 4.3 \\
\hline
\end{tabular}

has been used in earlier work for comparing sampling strategies [25], [26], and has shown robust performance in extensive experiments. Based on earlier experimental results (see, e.g., [25] and [26]) and our own experiences, we have chosen $D$ to be 0.25 and $K$ to be 20 . We have intentionally chosen slightly lower values so that our planner does not get unfair advantages.

We also ran the same experiments with two other sampling strategies, the uniform sampler and pure RBB (without mixing with uniform sampling). The uniform sampler is used mainly as a way to calibrate the difficulty of the queries, and RBB is used to examine the benefit of hybrid sampling. For RBB, the standard deviation $\sigma$ of the Gaussian is set to $1 / 8$. Without the help from the uniform sampler, we cannot make $\sigma$ too small. Otherwise, it would reduce the coverage of the roadmap and adversely affect the performance of the pure RBB.

For comparison, we also experimented with the Gaussian sampler and visibility-based PRM (Vis-PRM), with optimized parameter settings. In particular, for the Gaussian sampler, we varied the standard deviation of the Gaussian, using five different settings between $1 / 512$ and $1 / 16$, and chose the best one for each environment.

We ran each sampling strategy 30 times independently for each test environment, and terminated the planner as soon as a path was found between query configurations. The average number of milestones in the final roadmap, $N_{\text {mil }}$, and the average running time are shown in Table I. The statistics were gathered from the Java implementation of our planner on a Linux workstation with a $2.8 \mathrm{GHz}$ Pentium 4 processor.

Table I shows consistent results in these tests with different robots and environments. Hybrid sampling usually outperforms
TABLE II

BREAKDOWN OF TOTAL RUNNING TIME INTO COMPONENTS DEFINED IN SECTION III FOR THE EXAMPLE IN Fig. 10. $T_{\text {mil }}$ AND $T_{\text {con }}$ ARE MEASURED IN THE NuMBER OF CALls TO CleARANCE

\begin{tabular}{lrrrrr}
\hline Sampler & \multicolumn{1}{c}{$N_{\text {mil }}$} & \multicolumn{1}{c}{$T_{\text {mil }}$} & \multicolumn{1}{c}{$N_{\text {con }}$} & $T_{\text {con }}$ & Time (sec.) \\
\hline Hybrid & 1,004 & 113.5 & 7,180 & 43.3 & 41.4 \\
RBB & 4,155 & 33.4 & 11,127 & 59.0 & 98.2 \\
Uniform & 35,822 & 1.8 & 50,315 & 66.2 & $1,803.3$ \\
Visibility & 620 & 10825.2 & 4,955 & 93.9 & 698.1 \\
Gaussian & 3,255 & 18.5 & 15,699 & 46.4 & 92.2 \\
\hline
\end{tabular}

both the uniform sampler and pure RBB. Its roadmap is much smaller (see column 4), and the total running time is also shorter. The reduction in running time is not proportional to the reduction in roadmap size, because hybrid sampling pays a higher cost to obtain a milestone than the simpler uniform sampling. However, the smaller roadmap size requires much fewer tests to check collision-free connections between the milestones in the roadmap (see Table II), which are the dominant factor in the total running time. So hybrid sampling is able to achieve good overall performance. This basically confirms our intuition on the benefit of using the bridge test, as described in Section III.

For hybrid sampling, the standard deviation $\sigma$ used for the bridge test does affect the performance, sometimes by as much as $50 \%$. Nevertheless, hybrid sampling performs better than uniform sampling and RBB in all the environments with narrow passages, as long as a reasonable, not necessarily the best, value for $\sigma$ is chosen. This shows the benefit of hybrid sampling, even if we do not know the best value for $\sigma$.

In most of these experiments, RBB alone without uniform sampling does not perform as well as hybrid sampling, because these environments contain both narrow passages and wideopen free space. Without uniform sampling, we have to set $\sigma$ for the bridge test to a relatively large value in order to improve the coverage of the roadmap. As a result, the ability of the bridge test to identify narrow passages is reduced, leading to worse performance.

For Vis-PRM and the Gaussian sampler, as well as our hybrid sampling strategy, they all try to reduce the size of the roadmap and improve computational efficiency by filtering out milestones that are not useful. Yet they differ in the filtering cost (see Table II, column 3 for an example). To certify a useful milestone, Vis-PRM must perform several tests to check collisionfree connection between milestones, a very expensive operation. The cost of filtering for both Gaussian sampling and hybrid sampling is much cheaper. Gaussian sampling takes two calls to CLEARANCE to certify a useful milestone, and RBB takes three calls. As a result, although Vis-PRM produces very small roadmaps (see Table II, column 2) and clearly improves over uniform sampling, its performance is weaker than the Gaussian sampler and the hybrid sampling strategy using RBB. This is expected, in light of our discussion in Section III, and is why we suggest that $T_{\text {mil }}$ must remain small for the filtering to be effective. Gaussian sampling assumes that useful milestones lie close to obstacle boundaries. When this assumption holds, it has an advantage over hybrid sampling (see the example in Fig. 6). When the assumption fails, it may be substantially slower than hybrid sampling, especially for robots with many DOFs, shown in the example in Fig. 10. 

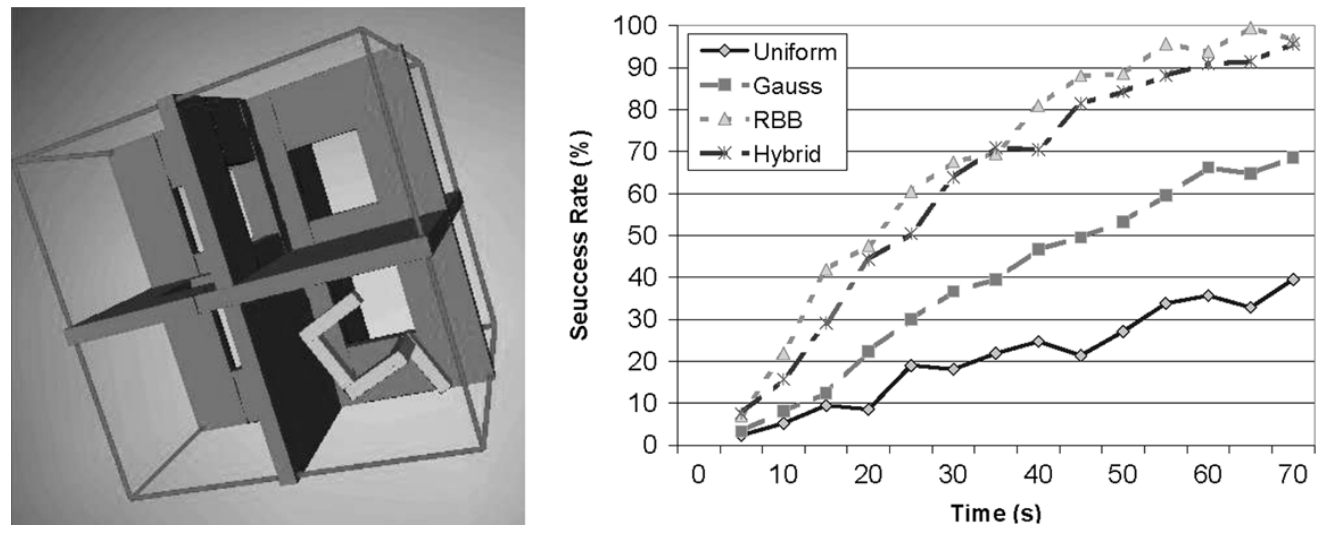

Fig. 11. Rigid body translating and rotating freely in a 3-D environment.
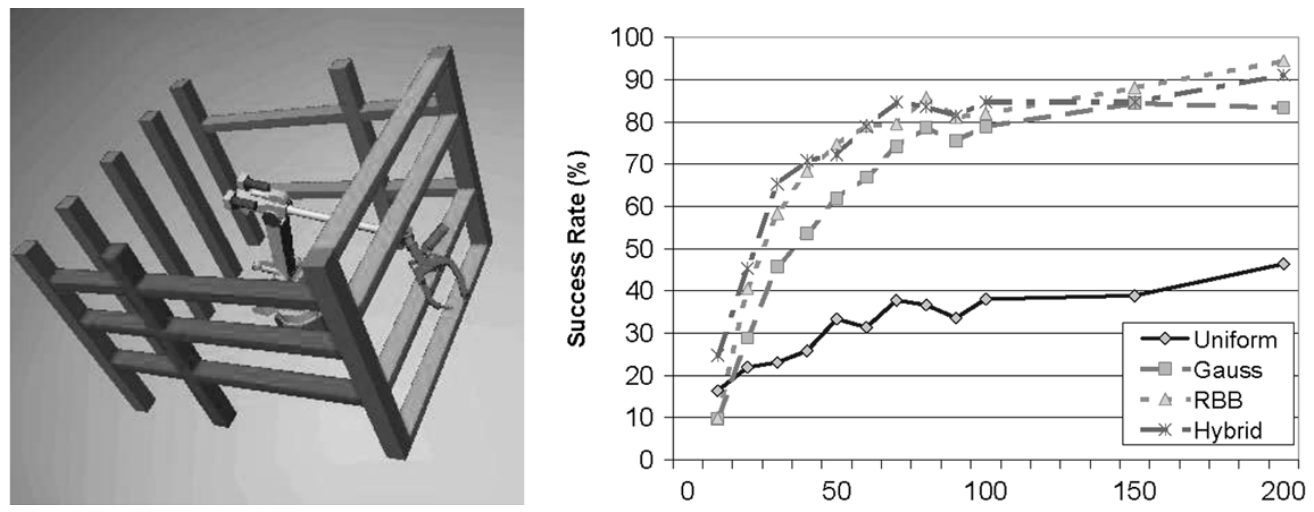

Fig. 12. 6-DOF robot manipulator.

The first five environments in Table I share a common characteristic: they all contain narrow passages connecting regions that allow relatively unrestricted movement. This gives an advantage to sampling strategies designed specifically for narrow passages, such as the hybrid sampling strategy or RBB. The environment in Fig. 6 is different. It contains a long path that has almost equal width everywhere. The resulting roadmaps all have roughly the same size, so the uniform sampler, which has low cost for sampling a milestone, performs better. However, even in this case, hybrid sampling performs only $9 \%$ worse than uniform sampling.

We also tested the $\mathrm{C}++$ implementation of our planner in 3-D environments. For each environment, we manually specified several queries, so that solving these queries indicates that a roadmap captures the connectivity of the free space well. Again, each test was repeated 30 times independently. For each test environment below, we show a graph that plots the percentage of queries that a planner can answer correctly as the running time increases.

- Fig. 11: This test uses a rigid-body robot translating and rotating freely in a 3-D environment. The space is divided into eight chambers by walls with holes. We specified query configurations in all the chambers, and ran the planners until all possible connections are established among the query configurations. The configuration space here consists of many narrow passages, with a moderate amount of free space that allows unrestricted movement.
Pure RBB turns out to have the best performance, because it focuses on sampling in narrow passages. The performance of the hybrid sampling strategy is close.

- Fig. 12: Here we have a 6-DOF robot manipulator arm. Horizontal and vertical bars are set up around the robot to make its movement difficult. We specified 12 queries. Each query requires the robot to move its end-effector from one opening between the bars to another. To answer a query, the robot must pull its end-effector out of a narrow opening, move in relatively open free space, and reinsert the end-effector into another narrow opening. In this test, the hybrid sampling strategy, RBB, and the Gaussian sampler have similar performance, all significantly better than uniform sampling.

\section{DISCUSSION}

Compared with the idea of sampling near obstacle boundaries, the bridge test gains efficiency by filtering out those samples near uninteresting obstacle boundaries, but it is not perfect. Some uninteresting samples near corners can pass the bridge test, because near the tip of a corner, it is easy to build short bridges. Fig. 5(c) shows that RBB generated a number of milestones near the corners of $\mathcal{F}$, and many of these milestones may be unhelpful. The bridge test generates false positives in this case, because it is a test of local geometry. As Fig. 13 shows, if we only have information within a small neighborhood, we cannot tell the difference between a narrow passage 

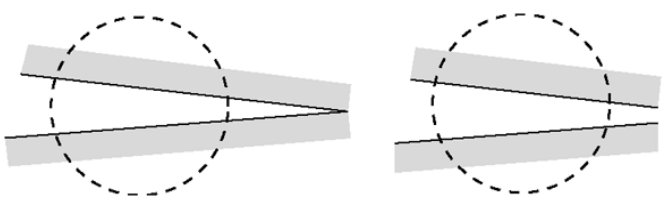

Fig. 13. Narrow passage and a narrow dead-end. Within the small neighborhood marked by the dashed circles, there is not enough information to differentiate these two cases.

and a narrow dead-end. Despite this problem, our experiments show that the benefits gained by sampling in narrow passages usually outweigh the computation time wasted in sampling near corners (see Section VII).

We can try to reduce the false positives near corners by taking additional samples. For example, the orthogonal test picks at random an additional segment $s^{\prime}$ through the configuration $q$, such that the segment $s^{\prime}$ is orthogonal to the bridge $s$. We accept $q$ as a new milestone if the endpoints of $s^{\prime}$ are both in collision or both free, in addition to the normal conditions on $s$. This helps to reduce the false positives, because such samples often have one endpoint in collision and one endpoint free. However, the orthogonal test is more expensive: it takes five calls to CLEARANCE per test in the worst case, while the original bridge test takes only three.

What is in common among the orthogonal test, the bridge test, and the Gaussian sampler is that they all try to reconstruct the local geometry of the configuration space by taking a small number of samples. We can think of other tests based on local sampling, as well. The more samples we take, the more likely that we can reconstruct the geometry accurately, at least in principle; at the same time, we have to pay a higher cost for sampling. In the extreme case, we can also check the collision-free connection between two sampled points, as Vis-PRM does. This yields very accurate information about the connectivity of the local free space, but it is very expensive. Our experiments indicate that the filtering cost should remain low in order for it to be effective.

\section{CONCLUSION AND Future WORK}

We have presented a hybrid sampling strategy to address the narrow passage problem for PRM planning. A key ingredient of the new sampling strategy is the bridge test, which boosts the sample density in narrow passages. The bridge test can be viewed as a filter that rejects the milestones that are unlikely to improve the connectivity of a roadmap, and thus saves the computation time by avoiding the expensive tests needed to connect these milestones in the roadmap. The bridge test is purely local, and can be implemented efficiently in high-dimensional configuration spaces. By combining the bridge test with uniform sampling, we construct a hybrid sampling strategy that generates small roadmaps that cover the free space well and have good connectivity. Our experiments on rigid and articulated robots in 2-D and 3-D environments show that our planner was able to reliably capture the connectivity of free spaces with difficult narrow passages.

There are two main issues that we are interested in exploring further in the future. First, in our current hybrid sampling strategy, the weight for combining the bridge test and the uniform sampler is set manually, and the best choice is clearly problem-dependent. A promising approach is to adjust the weights adaptively through online learning. It would also be interesting exploit the possibly complementary strengths of RBB and the Gausssian sampler, and combine them, as well as the uniform sampler, in a hybrid sampling framework. Work is currently underway in this direction [28].

Second, viewing the bridge test as a filter allows us to combine it with other sampling strategies. For example, MAPRM [22] samples points on the medial axis of $\mathcal{F}$. Since the milestones on the medial axis of the wide-open regions of $\mathcal{F}$ tend to have good coverage of $\mathcal{F}$, removing some of them does not affect the connectivity of the roadmap. On the other hand, milestones on the medial axis of narrow passages are more critical, as they have much more limited visibility. Removing any of them may disconnect the roadmap. Therefore, after generating milestones using MAPRM, we can apply the bridge test to the milestones. Each milestone that passes the bridge test is retained; each one that fails is retained with a certain probability. This way, we can get a smaller roadmap without affecting its quality.

\section{ACKNOWLEDGMENT}

The authors thank J.-C. Latombe for many insightful comments, and the anonymous reviewers for suggestions that have helped strengthen the paper.

\section{REFERENCES}

[1] N. M. Amato, O. B. Bayazit, L. K. Dale, C. Jones, and D. Vallejo, "OBPRM: An obstacle-based PRM for 3D workspaces," in Proc. 3rd Workshop Algorithmic Found. Robot., 1998, pp. 155-168.

[2] R. Bohlin and L. E. Kavraki, "Path planning using lazy PRM," in Proc. IEEE Int. Conf. Robot. Autom., 2000, pp. 521-528.

[3] V. Boor, M. H. Overmars, and A. F. van der Stappen, "The Gaussian sampling strategy for probabilistic roadmap planners," in Proc. IEEE Int. Conf. Robot. Autom., 1999, pp. 1018-1023.

[4] L. K. Dale and N. M. Amato, "Probabilistic roadmaps-Putting it all together," in Proc. IEEE Int. Conf. Robot. Autom., 2001, pp. 1940-1947.

[5] M. Foskey, M. Garber, M. C. Lin, and D. Manocha, "A Voronoi-based hybrid motion planner," in Proc. IEEE/RSJ Int. Conf. Intell. Robots Syst., 2001, pp. 55-60.

[6] D. Hsu, J.-C. Latombe, and R. Motwani, "Path planning in expansive configuration spaces," Int. J. Computat. Geom. Applic., vol. 9, no. 4-5, pp. 495-512, 1999.

[7] L. E. Kavraki, P. Švestka, J.-C. Latombe, and M. H. Overmars, "Probabilistic roadmaps for path planning in high-dimensional configuration spaces," IEEE Trans. Robot. Autom., vol. 12, no. 4, pp. 566-580, Aug. 1996.

[8] S. M. LaValle and J. J. Kuffner, "Randomized kinodynamic planning," Int. J. Robot. Res., vol. 20, no. 5, pp. 278-400, 2001.

[9] S. R. Lindemann and S. M. LaValle, "Incremental low-discrepancy lattice methods for motion planning," in Proc. IEEE Int. Conf. Robot. Autom., 2003, pp. 2920-2927.

[10] C. Nissoux, T. Siméon, and J.-P. Laumond, "Visibility-based probabilistic roadmaps," in Proc. IEEE/RSJ Int. Conf. Intell. Robots Syst., 1999, pp. 1316-1321.

[11] N. M. Amato, K. A. Dill, and G. Song, "Using motion planning to map protein folding landscapes and analyze folding kinetics of known native structures," in Proc. 6th Annu. Int. Conf. Computat. Biol., 2002, pp. 2-11.

[12] M. S. Apaydin, D. L. Brutlag, C. Guestrin, D. Hsu, and J.-C. Latombe, "Stochastic roadmap simulation: An efficient representation and algorithm for analyzing molecular motion," in Proc. 6th Annu. Int. Conf. Computat. Biol., 2002, pp. 12-21.

[13] H. Chang and T.-Y. Li, "Assembly maintainability study with motion planning," in Proc. IEEE Int. Conf. Robot. Autom., 1995, pp. 1012-1019. 
[14] T. Siméon, J.-P. Laumond, C. Geem, and J. Cortes, "Computer-aided motion: Move3D within MOLOG," in Proc. IEEE Int. Conf. Robot. Autom., 2001, pp. 1494-1499.

[15] A. P. Singh, J.-C. Latombe, and D. L. Brutlag, "A motion planning approach to flexible ligand binding," in Proc. 7th Int. Conf. Intell. Syst. Molecular Biol., 1999, pp. 252-261.

[16] J. H. Reif, "Complexity of the mover's problem and generalizations," in Proc. 20th IEEE Symp. Found. Computer Sci., 1979, pp. 421-427.

[17] J. H. Reif and Z. Sun, "On frictional mechanical systems and their computational power," SIAM J. Comput., vol. 32, no. 6, pp. 1449-1474, 2003

[18] J. Sellen, "Lower bounds for geometrical and physical problems," SIAM J. Comput., vol. 25, no. 6, pp. 1231-1253, 1996.

[19] J. Barraquand, L. E. Kavraki, J.-C. Latombe, T. Li, R. Motwani, and P. Raghavan, "A random sampling scheme for path planning," Int. J. Robot. Res., vol. 16, no. 6, pp. 759-774, 1997.

[20] D. Hsu, L. E. Kavraki, J.-C. Latombe, R. Motwari, and S. Sorkin, "On finding narrow passages with probabilistic roadmap planners," in Proc. 3rd Workshop Algorithmic Found. Robot., 1998, pp. 141-153.

[21] A. D. Collins, P. K. Agarwal, and J. L. Harer, "HPRM: A hierarchical PRM," in Proc. IEEE Int. Conf. Robot. Autom., 2003, pp. 4433-4438.

[22] S. A. Wilmarth, N. M. Amato, and P. F. Stiller, "Motion planning for a rigid body using random networks on the medial axis of the free space," in Proc. 15th Annu. ACM Symp. Computat. Geom., 1999, pp. 173-180.

[23] C. Holleman and L. E. Kavraki, "A framework for using the workspace medial axis in PRM planners," in Proc. IEEE Int. Conf. Robot. Autom., 2000, pp. 1408-1413.

[24] Y. Yang and O. Brock, "Adapting the sampling distribution in PRM planners based on an approximated medial axis," in Proc. IEEE Int. Conf. Robot. Autom., vol. 5, 2004, pp. 4405-4410.

[25] R. Geraerts and M. H. Overmars, "A comparative study of probabilistic roadmap planners," in Algorithmic Foundations of Robotics $V$. New York: Springer, 2002, pp. 43-59.

[26] — "Sampling techniques for probabilistic roadmap planners," in Proc. 8th Conf. Intell. Auton. Syst., 2004, pp. 600-609.

[27] D. Hsu and Z. Sun, "Adaptively combining multiple sampling strategies for probabilistic roadmap planning," in Proc. IEEE Conf. Robot., Autom., Mechatron., 2004, pp. 774-779.

[28] D. Hsu, G. Sánchez-Ante, and Z. Sun, "Hybrid PRM sampling with a cost-sensitive adaptive strategy," in Proc. IEEE Int. Conf. Robot. Autom., 2005, pp. 3885-3891

[29] M. Morales, L. Tapia, R. Pearce, S. Rodriguez, and N. M. Amato, "A machine learning approach for feature-sensitive motion planning," in Algorithmic Foundations of Robotics VI. Berlin, Germany: SpringerVerlag, 2004, pp. 361-376.

[30] S. Quinlan, "Efficient distance computation between nonconvex objects," in Proc. IEEE Int. Conf. Robot. Autom., 1994, pp. 3324-3330.

[31] S. Gottschalk, M. Lin, and D. Manocha, "OBBTree: A hierarchical structure for rapid interference detection," in Proc. 23rd Аnпи. Conf. Computer Graphics Interactive Tech., 1996, pp. 171-180.

[32] J.-C. Latombe, Robot Motion Planning. Boston, MA: Kluwer, 1991.

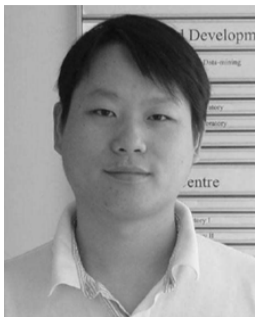

Zheng Sun (M'04) received the B.S. degree in computer science from Fudan University, Shanghai, China, in 1995, and the M.S. and Ph.D. degrees in computer science from Duke University, Durham, NC, in 1998 and 2003, respectively.

From 1999 to 2002, he was with Microsoft Corporation and Perfect Commerce as a Software Developer. In 2003, he joined the faculty of Hong Kong Baptist University, Kowloon, where he is an Assistant Professor of Computer Science. His research interests include robotic motion planning, computational geometry, and data mining and its applications in e-Commerce.

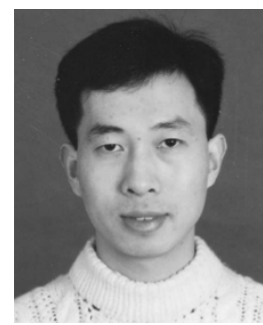

David Hsu received the B.Sc. degree in computer science and mathematics from the University of British Columbia, Vancouver, BC, Canada, in 1995, and the $\mathrm{Ph} . \mathrm{D}$. degree in computer science from Stanford University, Stanford, CA, in 2000

Currently, he is the Sung Kah Kay Assistant Professor of Computer Science with the National University of Singapore, and a Fellow of the Singapore-MIT Alliance. From 2000 to 2001, he was a Member of Research Staff with Compaq Computer Corporation's Cambridge Research Lab, Cambridge, MA. His main research interests include robotics, motion planning, computational biology, and geometric algorithms.

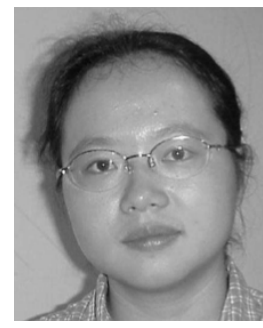

Tingting Jiang received the B.S. degree in computer science from the University of Science and Technology of China, Hefei, China, in 2001. She is currently working toward the Ph.D. degree in computer science at Duke University, Durham, NC.

Her current research interests are in computer vision, machine learning, and algorithms.

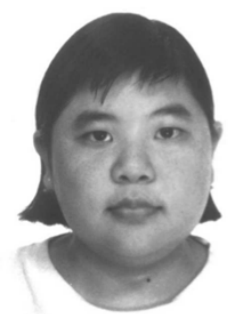

Hanna Kurniawati received the B.Sc. degree in computer science from the University of Indonesia, Depok, Indonesia, in 2001. She is currently working toward the Ph.D. degree in computer science at the National University of Singapore.

Her current research interests are robotics, motion planning, machine learning, and algorithms.

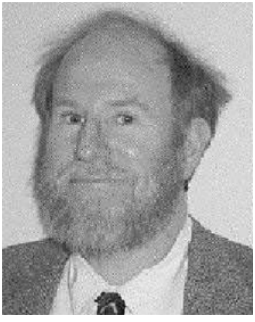

John H. Reif (S'77-M'78-SM'92-F'93) received the M.S. and Ph.D. degrees in applied mathematics in 1975 and 1977, respectively, from Harvard University, Cambridge, MA, and the B.S. degree (magna cum laude) in applied math and computer science in 1973 from Tufts University, Medford, MA

He has been the Hollis Edens Distinguished Professor in the Trinity College of Arts and Sciences at Duke University, Durham, NC, since 2003, and Professor of Computer Science at Duke University since 1986. Previously he was an Associate Professor with Harvard University. Although originally primarily a theoretical computer scientist, he also has made a number of contributions to practical areas of computer science, including parallel architectures, data compression, robotics, and optical computing. He has also worked for many years on the development and analysis of parallel algorithms for various fundamental problems, including the solution of large sparse systems, sorting, graph problems, data compression, etc. He has developed algorithms and lower bounds for a large variety of robotic motion-planning problems, and provided the first known computational complexity results for a robotic motion-planning problem. He has also developed a wide range of efficient parallel algorithms, particularly randomized parallel algorithms. Recently, he has worked on DNA computing and DNA nanostructures. He is the author of over 200 papers and has edited three books on synthesis of parallel and randomized algorithms.

Dr. Reif has been a Fellow of Association for the Advancement of Science (AAAS) since 2003, a Fellow of the Association of Computing Machinery (ACM) since 1996, and a Fellow of the Institute of Combinatorics since 1991. 